\title{
Spontaneous ventilation video-assisted thoracic surgery for mediastinal tumor resection in patients with pulmonary function deficiency
}

\author{
Weizhe Huang ${ }^{1}$, Hongsheng Deng ${ }^{2}$, Yuting $\mathrm{Lan}^{3}$, Runchen Wang ${ }^{4}$, Fan $\mathrm{Ge}^{4}$, Zhenyu Huo ${ }^{4}, \mathrm{Yi} \mathrm{Lu}^{4}$, \\ Weiyi Lin ${ }^{4}$, Guo Lin ${ }^{5}$, Wenhua Liang ${ }^{2}$, Hengrui Liang ${ }^{2}$, Jianxing $\mathrm{He}^{2}$
}

${ }^{1}$ Department of Thoracic Surgery, the First Affiliated Hospital of Shantou University Medical College, Shantou, Guangdong, China; ${ }^{2}$ Department of Thoracic Surgery and Oncology, the First Affiliated Hospital of Guangzhou Medical University, State Key Laboratory of Respiratory Disease, National Clinical Research Center for Respiratory Disease, Guangzhou Institute of Respiratory Health, Guangzhou, China; ${ }^{3}$ Mental Health College, Guangzhou Medical University, Guangzhou, China; ${ }^{4}$ Nanshan College, Guangzhou Medical University, Guangzhou, China; ${ }^{5}$ The First Clinical College, Guangzhou Medical University, Guangzhou, China

Contributions: (I) Conception and design: H Liang, W Huang; (II) Administrative support: J He; (III) Provision of study materials or patients: H Liang, W Huang; (IV) Collection and assembly of data: H Liang, W Liang; (V) Data analysis and interpretation: H Liang, W Huang; (VI) Manuscript writing: All authors; (VII) Final approval of manuscript: All authors.

Correspondence to: Wenhua Liang; Hengrui Liang; Jianxing He. Department of Thoracic Surgery and Oncology, the First Affiliated Hospital of Guangzhou Medical University, State Key Laboratory of Respiratory Disease, National Clinical Research Center for Respiratory Disease, Guangzhou Institute of Respiratory Health, Guangzhou, China. Email: liangwh1987@163.com; Hengrui_liang@163.com; drjianxing.he@gmail.com.

Background: Whether non-intubated spontaneous ventilation video-assisted thoracoscopic surgery (SVVATS) is a safe procedure remains controversial for mediastinal tumor patients with impaired lung function. Herein, we assessed feasibility of SV-VATS in lung function deficiency patients underwent mediastinal tumor resection.

Methods: From December 2015 to February 2020, 32 mediastinal tumor patients with impaired lung function (preoperative forced expiratory volume in 1 second $<70 \%$ of the predicted value) were retrospectively collected. Patients were divided into two groups: SV-VATS group and mechanical ventilation VATS (MV-VATS) group. Intraoperative and postoperative variables were compared between two cohorts.

Results: Fifteen patients (46.88\%) underwent SV-VATS and 17 patients $(53.12 \%)$ were performed with MV-VATS. The most common causes of lung function deficiency were smoking $(81.25 \%)$ and COPD (71.88\%). Patients in the SV-VATS group had similar blood loss (20.63 vs. $18.76 \mathrm{~mL}, \mathrm{P}=0.417)$ with MVVATS group. The anesthesia time (217.51 vs. $197.76 \mathrm{~min} ; \mathrm{P}=0.343)$ and surgery time (141.23 vs. $132.36 \mathrm{~min}$; $\mathrm{P}=0.209)$ were also similar between groups. Five people suffered postoperative complications in each group, in which 1 patient underwent MV-VATS was transferred to intensive care unit (ICU) because of prolonged extubation owing to hypoxia. There was no difference on chest tube removal time (2.6 vs. 2.3 days; $\mathrm{P}=0.172$ ) or hospital duration (5.03 vs. 4.74 days; $\mathrm{P}=0.297$ ) in patients underwent SV-VATS and MV-VATS.

Conclusions: SV-VATS is safe and provides similar short-term results to MV-VATS for mediastinal tumor resection in patients with limited pulmonary function.

Keywords: Video assisted thoracoscopic surgery (VATS); mediastinal tumor; impaired pulmonary function; spontaneous ventilation; non-intubated thoracic surgery

Submitted Feb 17, 2020. Accepted for publication Aug 17, 2020.

doi: 10.21037/atm-20-1652

View this article at: http://dx.doi.org/10.21037/atm-20-1652 


\section{Introduction}

Mediastinal tumors are heterogeneous diseases, which are composed of both benign and malignant diseases (1). Surgery is currently the best treatment for mediastinal tumors (2-4). Reported researches have demonstrated the convenience and profit of video-assisted thoracoscopic surgery (VATS) for mediastinal tumors, either in benign tumor, or in malignant mediastinal masses (5).

For traditional mechanical ventilation VATS (MVVATS), intubated general anesthesia with one-lung ventilation has widely been regarded as mandatory. However, complications after intubated general anesthesia are non-negligible, which might include airway damage, barotrauma and airway ciliary injury, etc. $(6,7)$ Nonintubated anesthesia with spontaneous ventilation has been applied in VATS (SV-VATS) to prevent the adverse events caused by tracheal intubation, mechanical ventilation, and muscle relaxants (8).

Patients with impaired lung function or chronic obstructive pulmonary disease (COPD) has widely been considered as high-risk for pulmonary or mediastinal surgery. There is a high incidence of postoperative respiratory failure for these patients, which may hamper them from fast recovery after tumor resection. In recent expert consensuses, impaired lung function is also one of contraindications for SV-VATS or tubeless thoracic surgery $(9,10)$.

Although it is not a routine practice, several studies have made an attempt and demonstrated SV-VATS was a feasible technique for lung function deficiency patients underwent pulmonary surgery (11). However, whether would SV-VATS successfully adapt to mediastinal lesion resection in patients with pulmonary insufficiency still remains controversial. The aim of this study was to evaluate the short-term results of SV-VATS for mediastinal lesion in patients with impaired pulmonary function. We present the following article in accordance with the STROBE reporting checklist, available at http://dx.doi.org/10.21037/atm-20-1652 (12).

\section{Methods}

\section{Study design and patient inclusion}

The study was conducted in accordance with the Declaration of Helsinki (as revised in 2013). The study was approved by the institutional ethics committee of the first affiliated hospital of Guangzhou Medical University (2019-
SVA) and informed consent was taken from all the patients.

The data of patients with impaired lung function that underwent mediastinal tumor resection between December 2015 and February 2020 were retrospectively and consecutively identified through electronic medical records. The experimental group is SV-VATS patients and the control group is MV-VATS group. Pulmonary ventilation function test was accomplished to identify patients with impaired lung function. Computed tomography (CT) scan of all patients was performed to confirm the size and position of lesions by two independent radiologists before surgery. Patients were included if they met the following criteria: (I) preoperative forced expiratory volume in one second (FEV1) below $70 \%$ of predicted; (II) mediastinal lesion less than $10 \mathrm{~cm}$ and no obvious invasion to other organs; (III) American society of anesthesiologists (ASA) status class $<3$.

Exclusive criteria: (I) patients that have anticipated difficult airway management; (II) patients that have bleeding disorders; (III) patients that have persistent cough or high amount of airway secretion; (IV) patients that have obesity (body mass index $>30$ ).

\section{Anesthesia and surgery}

Vital signs were routinely monitored for patients after entering the operating room. Midazolam $0.06 \mathrm{mg} / \mathrm{kg}$ and atropine $0.01 \mathrm{mg} / \mathrm{kg}$ were administered 30 minutes before anesthesia induction.

The detailed anesthesia protocol for mediastinal resection has been reported by our team previously (13). Briefly, in MV-VATS group, a double lumen tracheal tube was inserted after anesthesia induction. Muscle relaxant (cisatracurium), analgesic agents (sufentanil and remifentanil) and sedative (propofol and dexmedetomidine or inhaled sevoflurane) were given to maintain anesthesia for patients during operation. In SV-VATS group, double-laryngeal mask was used in placed of tracheal tube after anesthesia induction. No muscle relaxant was used during entire operation process. Low dose of analgesic agents (sufentanil and remifentanil) and intravenous sedative (propofol and dexmedetomidine) were given for maintenance. Synchronized intermittent mandatory ventilation (SIMV) mode was used if patients lose spontaneous ventilation in temporary. Regional anesthesia including incision blockade, intercostal nerve blockade and vagus nerve blockade were performed for patients in SV-VATS group. 
Operation was completed through lateral approach. The detail surgery process was described elsewhere by our team (13). A $1.5 \mathrm{~cm}$ skin incisions was made in the third intercostal space along the middle axillary line, the fifth intercostal space along the anterior axillary line, and the seventh intercostal space along the posterior axillary line. The left and right phrenic nerves were then identified and separated from the pericardium, including the anterior mediastinal pleura, thymus, and surrounding fatty tissue. The region of the mediastinal tumor was separated at the right side from the right brachiocephalic vein, on the left side from the innominate vein, and on the dorsal side from the brachiocephalic artery and trachea. After that, the mediastinal tumor was removed.

To reduce the risk of mucosa injury, we usually dissect the mediastinal tumor with carefully grasping and traction. All specimens were safely removed via a specimen bag by enlarging the upper port. Any bleeding or air leak was managed by reinforcement sutures using 4/0 PROLENE (Ethicon, Somerville, NJ, USA) or application of sealants such as Biopaper (Datsing Bio-Tech Co Ltd., Beijing, China). After the operation, a chest tube was placed through the observation hole.

\section{Postoperative care}

Patients were sent back to ward after waking up in recovery room. Chest X-ray scan was performed at the same day after operation and another time before discharge. Drinking and meal intake were resumed after bowel sound returned to 5 per minute with no nausea or vomiting. Two groups of patients were discharged by the following standard: body $\mathrm{T}^{\circ}<38.0{ }^{\circ} \mathrm{C}, \mathrm{SaO}_{2}$ on room air $>95 \%$, no complications requiring in-hospital treatment, no respiratory infections, lung re-expansion $>70 \%$ after chest tube removal, ultrasound pleural effusion $<8 \mathrm{~cm}$.

\section{Data collection and statistical analyses}

The baseline characteristics, intra- and perioperative outcomes and complications, pathology information of the mediastinal lesion and postoperative outcomes were collected from the medical records. Duration and volume of chest tube, and the pain intensity data were collected from the nursing records. Postoperative variables included re-opening rate, 30-day morbidity and mortality, length of hospital stay, postoperative coagulation function and arterial blood gas index, lactic acid, postoperative analgesics.

Descriptive statistics were reported as median (range) or mean \pm standard deviation for continuous data, and as number (\%) for categorical data. Student $t$-tests for independent data. Categorical variables are given as a count and percentage of patients and compared with the $\chi^{2}$ or Fisher exact test. All tests were 2-sided, with an a-level of 0.05. SPSS software (SPSS version 25.0; IBM Corp, Armonk, NY, USA) were used for all statistical evaluations.

\section{Results}

\section{Patients characteristics}

From December 2015 to February 2020, 391 mediastinal tumor patients were consecutively enrolled in this analysis (Figure 1), of which $32(8.18 \%)$ patients were diagnosed as lung function deficiency. The mean age was $65.47 \pm 10.46$, which was much older than entire cohort of mediastinal tumor patients in our previous report (13). The most common causes of lung function deficiency were smoking history (81.25\%) and COPD (71.88\%). Among these patients, 15 (46.88\%) underwent SV-VATS and 17 (53.12\%) were performed with MV-VATS. Two (11.76\%) patients in MV-VATS group had lung surgery (lobectomy) history. The characters of enrolled patients were shown in Table 1 . All baseline characters were balanced between groups.

\section{Intraoperative outcomes}

No surgery or anesthesia related death or convert to open case occurred either in SV-VATS group or the MV-VATS group. Cases both in MV-VATS and SV-VATS achieved $\mathrm{R} 0$ resection. There was no case in SV-VATS need to be converted to MV-VATS. Nine (60.00\%) and 11 (64.71\%) patients were found to have pleural adhesions during operation in SV-VATS group and MV-VATS group, respectively. Patients in the SV-VATS group had similar intraoperative blood loss (20.63 vs. $18.76 \mathrm{~mL}, \mathrm{P}=0.417$ ) with MV-VATS group. The anesthesia time (217.51 vs. $197.76 \mathrm{~min} ; \mathrm{P}=0.343)$ and surgery time (141.23 vs. 132.36 min; $\mathrm{P}=0.209)$ were also similar between groups. One patient underwent MV-VATS was transferred to intensive care unit (ICU) because of extubation failure owing to hypoxia. Extubation was successfully performed after 2 days observed in ICU for that patient and noninvasive positive pressure ventilation (NIPPV) was then 


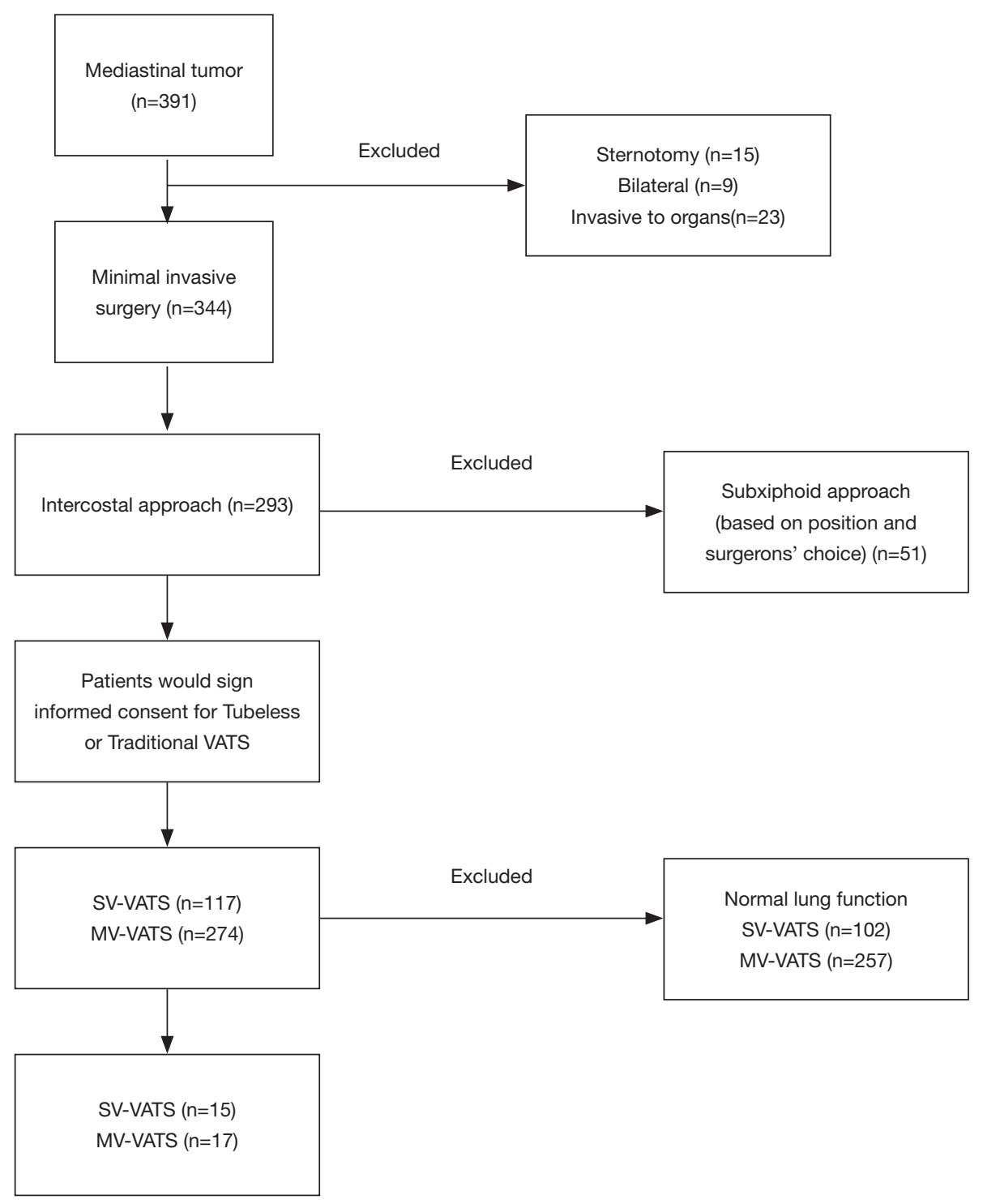

Figure 1 Flow chart of patient inclusion. SV-VATS, spontaneous ventilation video-assisted thoracic surgery; MV-VATS, mechanical ventilation video-assisted thoracic surgery.

used for 1 day before he left ICU.

\section{Postoperative recovery}

Five people suffered postoperative complications in each group. The detailed complications were summarized in Table 2. Although no statistical significance was observed, sore throat appeared more in MV-VATS group (11.8\% vs. $0 \%$ ).

The average of chest-tube duration was 2.6 and 2.3 days in SV-VATS and MV-VATS group $(\mathrm{P}=0.172)$, respectively. Average drainage volume was also similar between SV-
VATS and MV-VATS group (433 vs. $385 \mathrm{~mL}$; $\mathrm{P}=0.331$ ). Visual analogue scale (VAS) score was also similar in two groups $(2.43 \pm 0.89$ vs. $2.11 \pm 1.31 ; \mathrm{P}=0.174)$ on postoperative day 1 . In addition, there was no difference on hospital duration after surgery (5.03 vs. 4.74 days; $\mathrm{P}=0.297)$ in patients underwent SV-VATS and MV-VATS.

\section{Discussion}

VATS has been shown to be a safe way to treat mediastinal tumors, which is widely accepted due to the advantages of 
Table 1 Demographic characters of patients included

\begin{tabular}{|c|c|c|c|c|c|}
\hline Feature & SV-VATS (n=15) & $\mathrm{SD} / \%$ & MV-VATS $(n=17)$ & $\mathrm{SD} / \%$ & $\mathrm{P}$ \\
\hline Age (year) & & & & & 0.48 \\
\hline Mean & 63.90 & 11.76 & 67.43 & 14.40 & \\
\hline Male & 7 & $47 \%$ & 7 & $41 \%$ & \\
\hline Female & 8 & $53 \%$ & 10 & $59 \%$ & \\
\hline Mean & 22.01 & 3.67 & 23.43 & 2.25 & \\
\hline \multicolumn{2}{|c|}{ Preoperative lung function of predicted (\%) } & & & & 0.87 \\
\hline FVC & 76.7 & 11.2 & 74.3 & 13.6 & 0.43 \\
\hline $\mathrm{FEV}_{1}$ & 62.2 & 10.8 & 59.3 & 12.4 & 0.69 \\
\hline Non-smoker & 3 & $20 \%$ & 3 & $7 \%$ & \\
\hline Comorbidity & & & & & 0.77 \\
\hline Hypertension & 7 & $47 \%$ & 9 & $53 \%$ & \\
\hline Diabetes & 2 & $13 \%$ & 1 & $6 \%$ & \\
\hline Coronary heart disease & 2 & $13 \%$ & 3 & $18 \%$ & \\
\hline COPD & 10 & $67 \%$ & 13 & $76 \%$ & \\
\hline ASA status class & & & & & 0.62 \\
\hline I & 0 & $0 \%$ & 0 & $0 \%$ & \\
\hline II & 14 & $93 \%$ & 15 & $85 \%$ & \\
\hline Incision laterality & & & & & 0.49 \\
\hline Left & 8 & $53 \%$ & 7 & $41 \%$ & \\
\hline Right & 7 & $47 \%$ & 10 & $59 \%$ & \\
\hline Lesion length (cm) & & & & & 0.55 \\
\hline Mean & 5.12 & 1.43 & 5.27 & 1.63 & \\
\hline Pathology & & & & & 0.68 \\
\hline Thymoma & 4 & $27 \%$ & 5 & $29 \%$ & \\
\hline Neurogenic tumors & 2 & $14 \%$ & 1 & $6 \%$ & \\
\hline Cysts & 4 & $27 \%$ & 3 & $18 \%$ & \\
\hline Teratoma & 1 & $7 \%$ & 0 & $0 \%$ & \\
\hline Fibroma & 4 & $27 \%$ & 8 & $47 \%$ & \\
\hline
\end{tabular}

SV-VATS, spontaneous ventilation video-assisted thoracic surgery; MV-VATS, mechanical ventilation video-assisted thoracic surgery; BMI, body mass index; FVC, forced vital capacity; $\mathrm{FEV}_{1}$, forced expiratory volume in 1 second; ASA, American Society of Anesthesiologists; $\mathrm{SD}$, standard deviation; COPD, chronic obstructive pulmonary diseases. 
Table 2 Postoperative complication in SV-VATS group and MV-VATS group

\begin{tabular}{|c|c|c|c|c|}
\hline Complication & SV-VATS $(n=15)$ & $\%$ & MV-VATS $(n=17)$ & $\%$ \\
\hline Vomiting & 0 & 0 & 2 & 11.8 \\
\hline Dizziness & 1 & 6.7 & 1 & 5.9 \\
\hline Sore throat & 0 & 0 & 2 & 11.8 \\
\hline Fever & 1 & 6.7 & 1 & 5.9 \\
\hline Incision pain & 3 & 20 & 3 & 17.6 \\
\hline Prolonged extubation & 0 & 0 & 1 & 5.9 \\
\hline Dyspnea & 0 & 0 & 1 & 5.9 \\
\hline
\end{tabular}

SV-VATS, spontaneous ventilation video-assisted thoracic surgery; MV-VATS, mechanical ventilation video-assisted thoracic surgery.

smaller incision, shorter hospital stays, less postoperative pain and bleeding and less damage to the lung function. Non-intubated anesthesia with spontaneous ventilation has been applied in VATS to further prevent the adverse events caused by traditional intubated and mechanical induced lung injury. In this study, we reported our initial experience of SV-VATS for mediastinal tumor in patients with impaired lung function. The intraoperative and postoperative recovery outcomes showed that SV-VATS is a feasible and safety procedure for mediastinal tumor patients with lung function deficiency.

Previous researches and case reports have investigated the spontaneous for lung cancer in patients with impaired pulmonary function, either through thoracotomy or VATS (14-16). Twenty-eight lung cancer patients underwent SVVATS with impaired lung function were included in study from Wang et al. (11), they reported 1 patient required conversion to intubated, 5 patients had air leaks more than 5 days, 6 patents had subcutaneous emphysema, 2 patients developed acute exacerbations COPD, and 1 patient had atrial fibrillation after surgery. However, no comparison group was set in this study. Lan et al. (17) reported the management of SV-VATS in secondary contralateral thoracic surgery; these patients also damaged in lung function because of first lung operation. The study indicated the patients who undergo a secondary contralateral thoracic surgery had intraoperative stable vital signs and did not increase postoperative complications. Different from pulmonary surgery, lung would not be resected during mediastinal tumor operation. In our study, no significant clinical difference was observed between SV-VATS and
MV-VATS, which indicated SV-VATS is technically feasible and safe for mediastinal tumor resection. It can be used for situation when MV-VATS is contraindicated, such as muscle relaxant intolerance because of large mediastinal mass (18).

Numerous type of thoracic procedures can be applied with non-intubated techniques (19). Reported study have demonstrated its fine clinical efficacy in thoracic surgery (20). The advantages of non-intubated VATS for mediastinal surgery include: (I) lower risk of intubationrelated airway trauma, residual neuromuscular blockade, ventilator-induced lung injury and irritable cough $(21,22)$; (II) no muscle relaxant (such as cis-atracurium) and reduced dose of analgesic contribute to less anesthesiarelated complication and better muscle recovery (23); (III) better recovery of digestive functions due to avoidance of postoperative gastrointestinal dysfunction caused by general intubated anesthesia (24); (IV) more efficient diaphragmatic movements potentially provide better ventilation/ perfusion (V/Q) matching compared to mechanically ventilated patient (25). Moreover, a decreased mental burden, enhanced patient satisfaction and an improved rate of utilization of existing health resources could also be expected.

Several limitations of non-intubated VATS should also be acknowledged. (I) prolonged spontaneous breathing may lead to hypoxia (defined as $\mathrm{SpO}_{2}<90 \%$ ), and hypercapnia (defined as $\mathrm{PaCO}_{2} \geq 60 \mathrm{mmHg}$ ); (II) conversion to general anesthesia with intubation may happen due to uncontrollable ventilation situation. Previous studies have reported that the conversion rate was about $0-10 \%(26)$; (III) aspiration may occur without protection of the 
endotracheal tube. Inadequate fasting, gastroesophageal reflux disease or hiatal hernia would cause this complication. Thus, the anesthetist should be skillful to control over all these complex conditions. In spite of these situation, non-intubated VATS for mediastinal tumor is of good application prospect.

Concerns should also be considered with the use of non-intubated VATS for specific patients with impaired pulmonary function. Our study shows that non-intubated VATS is technically feasible in selected patients with impaired pulmonary function undergoing mediastinal tumor surgery. It may be an alternative anesthetic and surgical approach when managing mediastinal tumor patients who are considered high-risk for intubated general anesthesia. For patients with COPD, airway hyperactivity may present with acute exacerbations and result in severe complications perioperatively (27). In addition, general anesthesia causing respiratory depression, reduced hypoxia reflex, respiratory muscle relaxation and bronchial contraction may be intolerable for patients with impaired lung function. Furthermore, damage to chest wall and large vessels might exacerbate the impaired lung function. Thus, the probability of postoperative respiratory failure is increased. Non-intubated anesthesia with spontaneous ventilation could lower the risk of it and avoid further airway irritation due to less dose of analgesic (15). Although hypoxemia may develop during SV-VATS, it is mostly transient and can be easily improved with supplemental oxygen via high-flow humidified oxygen. Another challenge is that surgery for patients with impaired pulmonary function is associated with higher incidences of postoperative pulmonary complications, including pneumonia, atelectasis, acute heart failure, arrhythmia, pulmonary embolism, etc. Though SV-VATS was reported to decrease the risk of postoperative pulmonary complications, it is necessary to have careful postoperative management, discharge criteria and the follow-up plan. We suggest that appropriate patient selection, accumulated experience by performing minor SV-VATS procedures, and conversion to intubated general anesthesia without hesitation are essential to reduce the risk of emergency intubation and complications.

We acknowledge several limitations in this study. Firstly, the small sample size limited its statistical power. Secondly, single-institution retrospective design might incur bias in record and patient selection. Thirdly, insufficient follow-up time limited this study to report long-term change of lung function after surgery. Last, vial sign and blood gas test of patients were not recorded during operation. Randomized prospective studies for SV-VATS in patients with impaired lung function are warranted to validate the evidence.

\section{Conclusions}

SV-VATS is safe and provides similar short-term results to MV-VATS for mediastinal tumor resection in patients with limited pulmonary function.

\section{Acknowledgments}

Funding: None.

\section{Footnote}

Reporting Checklist: The authors have completed the STROBE reporting checklist. Available at http://dx.doi. org/10.21037/atm-20-1652

Data Sharing Statement: Available at http://dx.doi. org/10.21037/atm-20-1652

Peer Review File: Available at http://dx.doi.org/10.21037/ atm-20-1652

Conflicts of Interest: All authors have completed the ICMJE uniform disclosure form (available at http://dx.doi. org/10.21037/atm-20-1652). JH serves as an unpaid editorial board member of Annals of Translational Medicine from Jun 2019 to May 2024. The other authors have no conflicts of interest to declare.

Ethical Statement: The authors are accountable for all aspects of the work in ensuring that questions related to the accuracy or integrity of any part of the work are appropriately investigated and resolved. The study was conducted in accordance with the Declaration of Helsinki (as revised in 2013). The study was approved by the institutional ethics committee of the first affiliated hospital of Guangzhou Medical University (2019-SVA) and informed consent was taken from all the patients.

Open Access Statement: This is an Open Access article distributed in accordance with the Creative Commons Attribution-NonCommercial-NoDerivs 4.0 International License (CC BY-NC-ND 4.0), which permits the noncommercial replication and distribution of the article with the strict proviso that no changes or edits are made and the 
original work is properly cited (including links to both the formal publication through the relevant DOI and the license). See: https://creativecommons.org/licenses/by-nc-nd/4.0/.

\section{References}

1. Serra Fortuny $M$, Gallego $M$, Berna L, et al. FDG-PET parameters predicting mediastinal malignancy in lung cancer. BMC Pulm Med 2016;16:177.

2. Rabiou S, Lakranbi M, Ghizlane T, et al. Which surgery for mediastinum tumor: Experience of the Department of thoracic surgery of CHU Hassan II of Fes. Rev Pneumol Clin 2017;73:246-52.

3. Peng G, Liu M, Luo Q, et al. Spontaneous ventilation anesthesia combined with uniportal and tubeless thoracoscopic lung biopsy in selected patients with interstitial lung diseases. J Thorac Dis 2017;9:4494-501.

4. Kimura K, Kanzaki R, Kimura T, et al. Long-Term Outcomes After Surgical Resection for Pleural Dissemination of Thymoma. Ann Surg Oncol 2019;26:2073-80.

5. Hwang SK, Park SI, Kim YH, et al. Clinical results of surgical resection of mediastinal teratoma: efficacy of video-assisted thoracic surgery. Surg Endosc 2016;30:4065-8.

6. Drake-Brockman TF, Ramgolam A, Zhang G, et al. The effect of endotracheal tubes versus laryngeal mask airways on perioperative respiratory adverse events in infants: a randomised controlled trial. Lancet 2017;389:701-8.

7. Neto AS, Hemmes SN, Barbas CS, et al. Association between driving pressure and development of postoperative pulmonary complications in patients undergoing mechanical ventilation for general anaesthesia: a meta-analysis of individual patient data. Lancet Respir Med 2016;4:272-80.

8. Yang SM, Wang ML, Hung MH, et al. Tubeless Uniportal Thoracoscopic Wedge Resection for Peripheral Lung Nodules. Ann Thorac Surg 2017;103:462-8.

9. He J, Liu J, Zhu C, et al. Expert consensus on spontaneous ventilation video-assisted thoracoscopic surgery in primary spontaneous pneumothorax (Guangzhou). Ann Transl Med 2019;7:518.

10. He J, Liu J, Zhu C, et al. Expert consensus on tubeless video-assisted thoracoscopic surgery (Guangzhou). J Thorac Dis 2019;11:4101-8.

11. Wang ML, Hung MH, Hsu HH, et al. Non-intubated thoracoscopic surgery for lung cancer in patients with impaired pulmonary function. Ann Transl Med 2019;7:40.
12. von Elm E, Altman DG, Egger M, et al. The Strengthening the Reporting of Observational Studies in Epidemiology (STROBE) Statement: guidelines for reporting observational studies. Int J Surg 2014;12:1495-9.

13. Liang H, Liu J, Wu S, et al. Nonintubated Spontaneous Ventilation Offers Better Short-term Outcome for Mediastinal Tumor Surgery. Ann Thorac Surg 2019;108:1045-51.

14. Wu CY, Chen JS, Lin YS, et al. Feasibility and safety of nonintubated thoracoscopic lobectomy for geriatric lung cancer patients. Ann Thorac Surg 2013;95:405-11.

15. Wang ML, Hung MH, Hsu HH, et al. Non-intubated thoracoscopic surgery for lung cancer in patients with impaired pulmonary function. Ann Transl Med 2019;7:40.

16. Szabó Z, Tanczos T, Lebak G, et al. Non-intubated anaesthetic technique in open bilobectomy in a patient with severely impaired lung function. J Thorac Dis 2018;10:E275-80.

17. Lan L, Jiang L, Zhang C, et al. Feasibility of Spontaneous Ventilation in Secondary Contralateral Thoracic Surgery. Med Sci Monit 2019;25:9085-93.

18. Hartigan PM, Ng JM, Gill RR. Anesthesia in a Patient with a Large Mediastinal Mass. N Engl J Med 2018;379:587-8.

19. Gonzalez-Rivas D, Bonome C, Fieira E, et al. Nonintubated video-assisted thoracoscopic lung resections: the future of thoracic surgery? Eur J Cardiothorac Surg 2016;49:721-31.

20. Zhang $\mathrm{K}$, Chen HG, Wu WB, et al. Non-intubated videoassisted thoracoscopic surgery vs. intubated video-assisted thoracoscopic surgery for thoracic disease: a systematic review and meta-analysis of 1,684 cases. J Thorac Dis 2019;11:3556-68.

21. Miñambres E, Burón J, Ballesteros MA, et al. Tracheal rupture after endotracheal intubation: a literature systematic review. Eur J Cardiothorac Surg 2009;35:1056-62.

22. Dos Santos CC, Slutsky AS. Invited review: mechanisms of ventilator-induced lung injury: a perspective. J Appl Physiol (1985) 2000;89:1645-55.

23. Murphy GS, Szokol JW, Avram MJ, et al. Postoperative residual neuromuscular blockade is associated with impaired clinical recovery. Anesth Analg 2013;117:133-41.

24. Visser K, Hassink EA, Bonsel GJ, et al. Randomized controlled trial of total intravenous anesthesia with propofol versus inhalation anesthesia with isofluranenitrous oxide: postoperative nausea with vomiting and economic analysis. Anesthesiology 2001;95:616-26. 
25. Liu YJ, Hung MH, Hsu HH, et al. Effects on respiration of nonintubated anesthesia in thoracoscopic surgery under spontaneous ventilation. Ann Transl Med 2015;3:107.

26. Shi Y, Yu H, Huang L, et al. Postoperative pulmonary complications and hospital stay after lung resection surgery: A meta-analysis comparing nonintubated and intubated anesthesia. Medicine (Baltimore) 2018;97:e10596.

27. Warner DO, Warner MA, Offord KP, et al. Airway obstruction and perioperative complications in smokers undergoing abdominal surgery. Anesthesiology 1999;90:372-9.

Cite this article as: Huang W, Deng H, Lan Y, Wang R, Ge F, Huo Z, Lu Y, Lin W, Lin G, Liang W, Liang H, He J. Spontaneous ventilation video-assisted thoracic surgery for mediastinal tumor resection in patients with pulmonary function deficiency. Ann Transl Med 2020;8(21):1444. doi: 10.21037/atm-20-1652 